\title{
Functional imaging reveals movement preparatory activity in the vegetative state
}

\section{Tristan Andres Bekinschtein ${ }^{1,2,3,4}$, Facundo Francisco Manes ${ }^{1,2,5}$, Mirta Villarreal ${ }^{2}$, Adrian Mark Owen ${ }^{3,4}$ and Valeria Della-Maggiore ${ }^{6}$}

\author{
1 Institute of Cognitive Neurology, Buenos Aires, Argentina \\ 2 Raúl Carrea Institute for Neurological Research, Buenos Aires, Argentina \\ 3 Impaired Consciousness Research Group, Addenbrooke's Hospital, University of Cambridge, Cambridge, UK \\ 4 Cognition and Brain Sciences Unit, Medical Research Council, Cambridge, UK \\ ${ }_{5}$ Institute of Neuroscience, Favaloro University, Buenos Aires, Argentina \\ 6 Department of Physiology, School of Medicine, University of Buenos Aires, Buenos Aires, Argentina
}

\section{Edited by:}

Patrik Vuilleumier, University Medical Center and University Hospital Geneva, Switzerland

\section{Reviewed by:}

Patrick Haggard, University College London, UK

Patrik Vuilleumier, University Medical Center and University Hospital Geneva, Switzerland

Steven Laureys, University of Liège, Belgium

\section{*Correspondence:}

Tristan Andres Bekinschtein, Cognition and Brain Sciences Unit, Medical Research Council, 15 Chaucer Road, Cambridge CB2 7EF, UK.

e-mail: tristan.bekinschtein@mrc-cbu. cam.ac.uk
The vegetative state (VS) is characterized by the absence of awareness of self or the environment and preserved autonomic functions. The diagnosis relies critically on the lack of consistent signs of purposeful behavior in response to external stimulation. Yet, given that patients with disorders of consciousness often exhibit fragmented movement patterns, voluntary actions may go unnoticed. Here we designed a simple motor paradigm that could potentially detect signs of purposeful behavior in VS patients with mild to severe brain damage by examining the neural correlates of motor preparation in response to verbal commands. Twenty-four patients who met the diagnostic criteria for VS were recruited for this study. Eleven of these patients showing preserved auditory evoked potentials underwent functional magnetic resonance imaging (fMRI) to test for basic speech processing. Five of these patients, who showed word related activity, were included in a second fMRI study aimed at detecting functional changes in premotor cortex elicited by specific verbal instructions to move either their left or their right hand. Despite the lack of overt muscle activity, two patients out of five activated the dorsal premotor cortex contralateral to the instructed hand, consistent with movement preparation. Our results may reflect residual voluntary processing in these two patients. We believe that the identification of positive results with $\mathrm{fMRI}$ using this simple task, may complement the clinical assessment by helping attain a more precise diagnosis in patients with disorders of consciousness.

Keywords: consciousness, intention, vegetative state, functional imaging, movement preparation

\section{INTRODUCTION}

The vegetative state (VS), a disorder often observed after traumatic brain injury, is characterized by the absence of awareness of self or the environment and preserved autonomic functions (MultiSociety Task Force on PVS, 1994; Royal College of Physicians, 2003). To date, the diagnosis of VS has relied on behavioral assessment, most importantly, the lack of consistent signs of purposeful behavior in response to external stimulation. Yet, due to severe diffuse brain damage (Adams et al., 2000) these patients often exhibit fragmented movement patterns which may hinder the discrimination between reflexive and voluntary behavior (Schiff et al., 2002). On the other hand, some VS patients may retain partial levels of conscious processing, but be unable to reproducibly perform movements due to lesions at one or more levels of the motor system (Bekinschtein et al., 2008). Relying on the absence of overt behavior as the diagnostic criterion for VS may thus render the clinical assessment imprecise and subjective, leading to high error rates (Childs et al., 1993; Andrews et al., 1996; Gill-Thwaites, 2006). There is currently a need for greater diagnostic precision for patients in states of impaired consciousness as the diagnosis may have an impact on prognosis and treatment (Owen and
Coleman, 2008); neurophysiological measures that may unveil voluntary responses to external stimuli should help to reduce the risk of misdiagnosis.

To date, only two studies have shown unequivocal, objective evidence of conscious awareness in clinically defined VS patients using functional magnetic resonance imaging (fMRI; Owen et al., 2006; Monti et al., 2010). Our group has previously demonstrated activation of two different functional networks when the patient is asked to imagine playing tennis (motor imagery) or walking throughout his/her apartment (spatial navigation; Owen et al., 2006). The brain pattern associated with these two tasks did not differ from that found in healthy volunteers. Nevertheless, the high cognitive demands associated with imagery (Guillot and Collet, 2005) may restrict its application to a subset of patients with relatively spared cognitive processing. In a recent study we demonstrated that, although movement may not be detectable in the behavioral assessment, it can be inferred from electromyographical recordings of hand muscles in response to a hand movement command (Bekinschtein et al., 2008). On this basis, it seems likely that there may be even more extreme cases where the motor system is insufficiently preserved to generate a movement. Here, we used a simple motor paradigm to objectively detect 
signs of residual purposeful behavior in VS patients by examining the neural correlates of movement preparation to command. We hypothesized that VS patients with residual voluntary processing would activate the dorsal premotor cortex contralateral to the specific hand command.

\section{MATERIALS AND METHODS PATIENT SCREENING}

An original sample of 24 patients fulfilling the following criteria for VS were initially considered for this study: (1) vegetative state; (2) age between 18 and 65 years; (3) systemically healthy; and (4) 4 months or more post injury. They underwent a thorough neurological and behavioral assessment including at least three Coma Recovery Scale-Revised (CRS-R; Giacino et al., 2004) evaluations before the fMRI scan and a neurophysiological assessment of the auditory system as a first screening step to ensure that auditory cortical responses were intact.

Auditory evoked potentials were preserved in 11 patients in at least one side; latencies were within the normal range suggesting functionally spared eighth cranial nerve and auditory nuclei in the pons and midbrain (American Neurophysiology Society, 2006). In a third screening test, these 11 patients were scanned with fMRI while passively listening to blocks of words or white noise (Bekinschtein, 2006) to test for preserved auditory processing of basic speech characteristics. Four of the 11 patients did not undergo further testing due to large fMRI artifacts associated with teeth grinding and/or excessive head movements. Of the remaining seven patients five showed word related activity in primary or secondary auditory cortex in at least one hemisphere, suggestive of preserved auditory processing of basic speech characteristics, and therefore were included as participants in the study. The hierarchical approach is depicted in Figure 1.

\section{ASSESSMENT OF ATROPHY}

We assessed the degree of cortical and subcortical atrophy using a visual rating scale based upon that developed by Galton et al. (2001). Briefly, we first defined atrophy levels from 0 to 4 using T1-3D anatomical images $(0=$ no atrophy, $1=$ very low, $2=$ mild, $3=$ severe, and $4=$ highly severe atrophy) in a group of 12 patients with neurodegenerative disorders, and applied the scale to the VS patients included in this study (see Table 1). Patients' T1-weighted images were assessed by two experienced raters (Tristan Andres Bekinschtein and Facundo Francisco Manes) who were blind to the patient's clinical history. Images were aligned and inspected using the free viewing software MRIcro for Windows (http:// www.psychology.nottingham.ac.uk/staff/cr1/mricro.htm) with no further transformations.

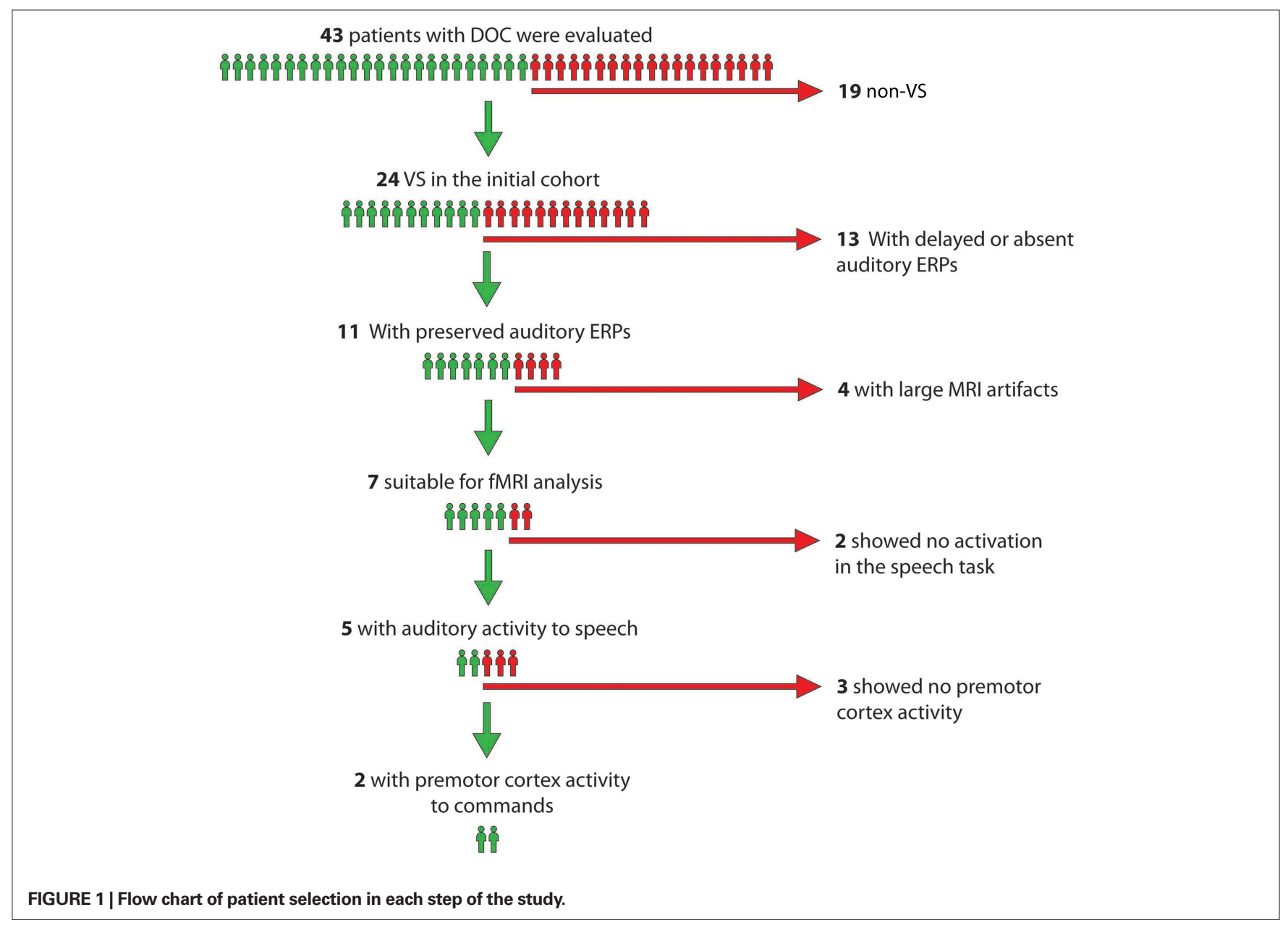




\section{PARTICIPANTS}

Signed assent from normal volunteers or patients' next of kin was obtained prior to participation. The Ethics and Research Committee of the Raul Carrea Institute approved the study. None of the patients' family withdrew assent. All 24 patients met the clinical diagnostic criteria defining the VS (Royal College of Physicians, 2003) and signs of awareness were checked to rule out cases of minimally conscious state (MCS) in agreement with the Aspen Neurobehavioral Conference Workgroup guidelines (Giacino et al., 2002). The five participants of this study included four patients with traumatic brain injury, of who two were due to car accidents (VS2 and VS5), one was due to a motorbike accident (VS1), and one due to a fall from a horse (VS4). The fifth patient (VS3) had mixed etiology (traumatic brain injury with anoxic events due to a delay of over $3 \mathrm{~h}$ before medical attention was received). Demographics and basic neurological and behavioral data are shown in Table 1. Further detailed description of each case can be found in Section "Patients' Assessments and Description" in Appendix. Data on three normal subjects who performed the same fMRI paradigm was also analyzed. The results, illustrating the normal task-related pattern of brain activity in premotor cortex are shown in Appendix.

\section{EXPERIMENTAL PROCEDURE}

Prior to entering the scanner, the five selected VS patients underwent an electromyographical assessment to evaluate spontaneous muscle activity and muscle subthreshold activity in response to a verbal instruction to move either their left or right hand (Bekinschtein et al., 2008). Muscle activity was measured using surface electrodes from the right and left flexor digitorum superficialis and abductor pollicis brevis muscles of the hand.

All five patients were then scanned with fMRI while being verbally instructed to move the right or the left hand. Instructions were delivered to all participants via headphones. We used a 24-s block design during which the same instruction was repeated six times (e.g., "Move your left hand"). There were a total of eight blocks for each hand command, and six commands per block. Blocks were separated by 15 -s silent periods (rest). Before entering each silent period, participants were instructed not to move the right or left hand, depending on the movement condition (e.g., "Now, do not move your left hand, stay still"). Given the lack of electromyographical equipment compatible with MRI, muscle activity was not quantified during the scanning session. However, as an additional control, we replicated the electromyographic measurement once the patients were removed from the scanner to make sure there were no signs of overt movement.

The three control subjects performed the same experimental paradigm and therefore, moved their hands in response to the commands.

\section{MRI ACOUISITION}

Functional images were acquired at the Raul Carrea Institute of Neurology on a 1.5-T GE Signa CVI MRI scanner, using T2*weighted echo planar image volumes with blood oxygen level dependent $(\mathrm{BOLD})$ contrast $(\mathrm{TR}=3000 \mathrm{~ms}, \mathrm{TE}=50 \mathrm{~ms}$, flip angle $=90^{\circ}, \mathrm{FOV}=240 \mathrm{~mm}$, gap $=0$ ). The 28 axial slices (in plane resolution $3.75 \times 3.75,5 \mathrm{~mm}$ thick) of each volume were aligned to the AC-PC line, covering the whole brain. The first five volumes of each series were discarded to allow for T2 saturation. After functional scanning, a T1-weighted anatomical image was obtained for each participant using a SPGR scanning protocol $(0.47 \mathrm{~mm} \times 0.47 \mathrm{~mm} \times 2.8 \mathrm{~mm})$. Scanner noise was continuous throughout the experiment providing a constant auditory background and attenuated with ear defenders and headphones. Head fixation was achieved by means of a foam cushion and by taping the subject's head to the head coil.

\section{DATA PRE-PROCESSING AND STATISTICAL ANALYSIS}

Functional images were pre-processed and analyzed using Statistical Parametric Mapping (SPM2, Wellcome Department of Cognitive Neurology, London, UK). Pre-processing steps included realignment and reslicing. Whole-brain univariate analyses were carried out for each individual subject by using a general lineal model for each voxel following a fixed-effects model. Data for each subject was modeled with a boxcar convolved with a synthetic hemodynamic response function. Movement parameters were entered as additional predictors in the general lineal model. A statistical voxel threshold of $p<0.001$ (uncorrected for multiple comparisons) with a minimum of three contiguous voxels was used for whole-brain analysis for three reasons: first, BOLD responses in this patient group are known to be diminished (Boly et al., 2004; Laureys et al., 2004), second, a relatively low magnetic field was used for this study (1.5 T), third, unlike most fMRI studies, in this study we sought to identify motor preparation responses at the single-subject level, not at a group level. In addition, region of interest (ROI) statistical analysis (small volume correction) was carried out to further assess

Table 1 | Demographic data, evoked potentials, behavioral scores, reflex and spontaneous movements for patient VS1 through VS5.

\begin{tabular}{|c|c|c|c|c|c|c|c|c|c|c|}
\hline Patient & $\begin{array}{l}\text { Time from } \\
\text { ictus (months) }\end{array}$ & Age & Etiology & Hemisphere & SSEP & BAEP & CRS-R & $\begin{array}{l}\text { Withdrawal } \\
\text { reflex }\end{array}$ & $\begin{array}{l}\text { Spontaneous } \\
\text { muscle activity }\end{array}$ & Brain atrophy \\
\hline VS1 & 16 & 24 & TBI & $L / R$ & $\mathrm{M} / \mathrm{N}$ & $\mathrm{N} / \mathrm{M}$ & 8 & $\mathrm{P} / \mathrm{P}$ & $\mathrm{P} / \mathrm{P}$ & 2 \\
\hline VS3 & 20 & 33 & TBI-anoxic & $L / R$ & $\mathrm{M} / \mathrm{N}$ & $\mathrm{N} / \mathrm{M}$ & 6 & $\mathrm{P} / \mathrm{P}$ & $\mathrm{P} / \mathrm{P}$ & 3 \\
\hline VS4 & 5 & 40 & TBI & $L / R$ & $\mathrm{M} / \mathrm{M}$ & $\mathrm{N} / \mathrm{S}$ & 4 & $\mathrm{~A} / \mathrm{P}$ & $\mathrm{A} / \mathrm{P}$ & 4 \\
\hline
\end{tabular}

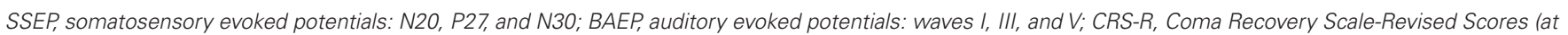

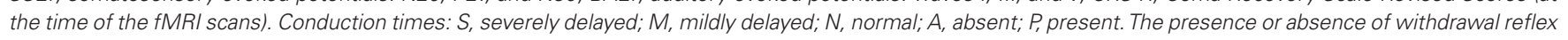
and spontaneous muscle activity follows the order of the Hemisphere column. 
the significance of premotor voxels using a more conservative criterion (Poldrack et al., 2008). Six different premotor ROIs were drawn following Picard and Strick's (2001)functional distinction using the MarsBar SPM toolbox (Brett et al., 2002). These are depicted in Figure 2, and correspond to right and left dorsal premotor cortex (PMd; this region lies on the most posterior part of the precentral gyrus), right and left pre-premotor cortex (pre-PMd, this region lies over the most anterior part of the precentral gyrus), supplementary motor area (SMA) proper and pre-SMA. MarsBar averages the beta values resulting from fitting the BOLD signal with the model for all voxels in each ROI, runs the GLM on this average and corrects for the total number of ROIs evaluated $(n=6)$. The ROI threshold was set at $p<0.05$ corrected for multiple comparisons. $t$ Values were adjusted by number or ROIs, defined a priori based on specific predictions drawn on our hypothesis stated in the introduction, not by total number of evaluated contrasts.

Functional images were pre-processed, registered to their corresponding T1 and manually normalized to the MNI anatomical template (MNI-152) using a linear transformation algorithm. ROIs were drawn on the MNI 152 template and superimposed onto each subject's structural image; final adjustments were performed manually to account for variability in gyri and sulci using the precentral gyrus, postcentral gyrus, and the central sulcus as anatomical landmarks.

\section{RESULTS}

\section{ELECTROMYOGRAPHICAL RECORDINGS}

All five patients showed spontaneous ballistic movement of the upper extremities and intact withdrawal reflexes to pressure in either the left or the right hands (Table 1). Electromyographical recordings were obtained before and after the scanning session while the same task was administered to rule out threshold and/ or subthreshold muscle activity associated with hand movement. Statistical analysis yielded no significant instruction-related activity for either the right or left hand.

\section{BRAIN ACTIVITY IN THE VEGETATIVE STATE Whole-brain analysis}

Hand movement command compared to rest. As a first step, we ran a whole-brain analysis examining global changes in motor and language related areas. All five VS patients showed lateral superior temporal cortex activity in response to auditory instructions, consistent with preserved auditory processing of basic speech characteristics. Auditory activation was highly variable (see Figure 3, columns 1 through 3). When hearing commands,

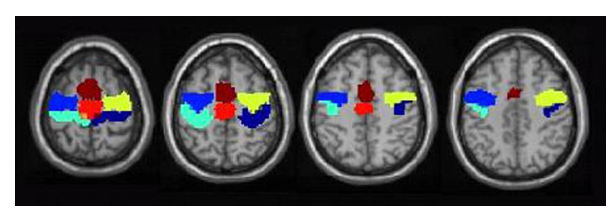

FIGURE 2 | Regions of interest for premotor cortex drawn based on Picard and Strick's (2001) functional distinction. Shown are the ROls drawn on the MNI-152 T1 template for right (dark blue) and left (light blue) PMd, right (yellow) and left (blue) pre-PMd, SMA proper (red) and pre-SMA (dark-red). patients VS1 and VS5 showed bilateral temporal-lobe activity while VS2, VS3, and VS4 showed activity only in the left temporal gyrus. In addition, activity in the middle temporal gyrus (MTG) was found in VS1 (medial and bilateral anterior MTG), VS2 (anterior and posterior MTG), and VS5 (posterior left MTG and medial right MTG) consistent with further semantic speech processing in neurologically intact subjects (Scott and Johnsrude, 2003). Besides the auditory activation, patients VS1 and VS2 showed greater activity in the left dorsal premotor cortex (see Figure 3, columns 4 through 6) in response to right-hand movement commands when compared to rest (VS1: $z=4.12$; $p<0.0005 ; 4$ voxels; and VS2: $z=3.67, p=0.0007 ; 10$ voxels $)$. In addition, VS2 showed activity in the SMA $(z=3.30 ; p=0.001$; 4 voxels) and the left dorsal pre-premotor cortex $(z=3.13$; $p=0.001 ; 3$ voxels). No activation was found for left-hand commands as compared to rest.

Specific hand command comparison. Whole-brain statistical analysis showed greater activity in the left dorsal premotor cortex $(\mathrm{z}=4.48, \mathrm{p}<0.0001 ; 6$ voxels; and $\mathrm{z}=3.92, \mathrm{p}=0.0006 ; 4$ voxels $)$ and the left inferior parietal lobe for VS1 $(\mathrm{z}=3.83, \mathrm{p}=0.0006 ; 5$ voxels) when right-hand movement commands were contrasted to left-hand movement commands in VS1. For the same contrast, VS2 showed greater activity in the left pre-premotor cortex $(\mathrm{z}=3.50$; $\mathrm{p}=0.0008)$, left inferior parietal lobe $(\mathrm{z}=3.48 ; \mathrm{p}=0.0008 ; 4$ voxels $)$, right superior parietal lobe $(\mathrm{z}=3.10 ; \mathrm{p}=0.001 ; 3$ voxels), and bilateral SMA ( $\mathrm{z}=4.43$; $\mathrm{p}<0.0001 ; 4$ voxels; Figure 4$)$. No significant differences were found when left-hand movement commands were compared to right-hand movement commands in either hemisphere.

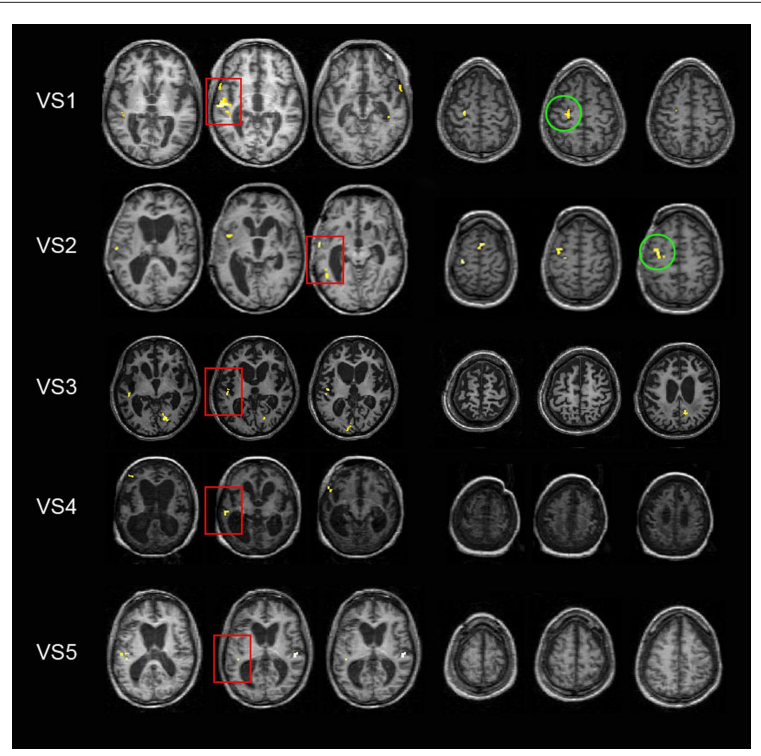

FIGURE 3 | Functional results from the whole-brain fixed-effect univariate analysis. Activation of temporal (red squares) and premotor cortex (green circles) is shown for patients VS1 through VS5 (top to bottom) when comparing right-hand commands vs. rest. All BOLD activity maps are superimposed on each individual's structural image. Statistical threshold level was set at $p<0.003$ for illustrative purposes. 


\section{Region of interest analysis}

To test the hypothesis that voluntary movement preparation would be reflected in greater BOLD activity in contralateral dorsal premotor cortex in response to a movement command, we ran a ROI statistical analysis using the masks for the dorsal premotor, pre-premotor, and SMAs depicted in Figure 2. Inferences were drawn at a $p$-value of 0.05 corrected for multiple comparisons (results are displayed in Table 2). Right vs. left-hand movement command yielded greater BOLD signal in the left dorsal premotor cortex for VS1 and VS2 $(\mathrm{VS} 1, t$ score $=2.60 ; p=0.029 ; \mathrm{VS} 2, t$ score $=2.75 ; p=0.020)$ and left pre-premotor cortex in VS2 ( $t$ score $=2.77 ; p=0.018)$. No significant activity was obtained for the other ROIs. Left vs. right-hand commands yielded no significant voxels for any of the ROIs. Figure 5 illustrates the time course of the BOLD signal in the left premotor cortex for both right vs. left and left vs. right-hand commands.

\section{DISCUSSION}

A wealth of neurophysiological and neuroimaging studies have demonstrated that movement preparation to externally triggered actions typically activates the dorsal premotor cortex (Wise et al., 1983; Passingham, 1985; Crammond and Kalaska, 1994, 2000; Toni et al., 1999; Thoenissen et al., 2002). Here, we showed that when instructed to move their right hand, two out of five vegetative patients with preserved auditory processing of basic speech characteristics, exhibited increased activity in the contralateral dorsal premotor cortex despite a lack of movement-related muscle activity. This predicted pattern of functional activation contralateral to the instructed hand movement strongly suggests movement preparation and/or planning, and thus, is consistent with a residual ability for conscious processing in these patients. Our findings are strengthened by the increment of activity in the contralateral posterior parietal cortex for both patients, a region involved in processing movement intention (Toni et al., 1999; Andersen and Buneo, 2002; Thoenissen et al., 2002).

It is important to remark that no significant activity was detected in premotor cortex in response to left-hand movement commands. This functional pattern may stem from anatomical lesions suffered during the accident or may be a consequence of subsequent atrophy or further tissue loss. The lack of activity for the left vs. right contrast in VS1 finds indirect support in his/her last behavioral assessment performed 34 months after the fMRI study: when pinched on the left arm, the patient crossed the body midline to reach it with his right arm but was unable to reach with the left

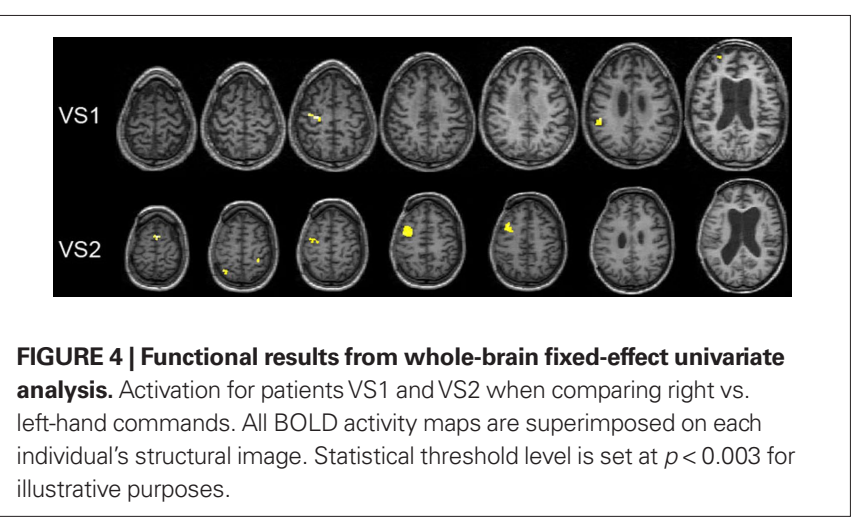

arm when pinched on the right arm. Despite showing spontaneous EMG activity for the muscles of the right arm, this patients' T1 image revealed severe callosal atrophy, commonly observed in TBI patients (e.g., Vuilleumier and Assal, 1995). Callosal atrophy may have interfered with interhemispheric processing affecting the right-hemisphere response to verbal commands of left-hand movements. As for VS2, the fact that no spontaneous EMG activity was detected for muscles of the right arm but withdrawal reflex was spared, suggests there was damage at the cerebral level of the motor (or sensory) system. The T1 image for this patient revealed no callosal damage, or any further atrophy in cortical motor/sensory cortex. This, however, does not rule out the existence of a cortical lesion that may have not been detectable at the low image resolution used (voxel size $=0.47 \mathrm{~mm} \times 0.47 \mathrm{~mm} \times 2.8 \mathrm{~mm})$. New MRI quantitative methods are now available to characterise the degree of tissue damage (Fernandez-espejo et al.,2011).

A number of previous studies have used neuroimaging methods to unveil cognitive processing in VS patients. The capacity to process painful stimuli (Laureys et al., 2002; Boly et al., 2008), auditory stimuli (Boly et al., 2004), some features of speech (Owen et al., 2002; Bekinschtein et al., 2004; Kotchoubey et al., 2005), and visual stimuli (Menon et al., 1998) has been reported in clinically diagnosed VS patients. More recently, Coleman et al. (2007, 2009a) identified a subset of VS patients ( 7 out of 22) who exhibited a pattern of brain activity consistent with speech processing. Moreover, our group also demonstrated conscious processing in 5 out of 54 patients with disorder of consciousness from which two truly VS patients, as determined by behavioral assessment, showed modulation of BOLD activity in response to an imagery task (Monti et al., 2010). Although, as revealed by fMRI, speech perception can occur in healthy subjects in some unconscious states such as sleep (Portas et al., 2000), recent evidence from anesthetized volunteers suggests that speech comprehension does require conscious awareness (Davis et al., 2007). In the context of this finding we suggest that VS1 and VS2 were able to process semantic characteristics of speech and used that information to activate movement-related networks.

Given the small number of patients with residual consciousness detected with $\mathrm{PMRI}$, one could argue that the technique is not efficient enough to implement as a standard routine in addition to

Table 2 | $p$ and $t$ values corresponding to "left vs. right" and "right vs. left" contrasts for VS1 and VS2 for each of the chosen ROls.

R-Pre-PMd R-PMd L-Pre-PMd L-PMd SMA Pre-SMA

\begin{tabular}{|c|c|c|c|c|c|c|}
\hline \multicolumn{7}{|c|}{ LEFT VS. RIGHT-HAND COMMANDS } \\
\hline VS1 & ns & ns & ns & ns & ns & ns \\
\hline VS2 & ns & ns & ns & ns & ns & ns \\
\hline \multicolumn{7}{|c|}{ RIGHT VS. LEFT-HAND COMMANDS } \\
\hline VS1 & ns & ns & $\begin{array}{l}p=0.020 \\
(t=2.75)\end{array}$ & $\begin{array}{l}p=0.029 \\
(t=2.60)\end{array}$ & ns & ns \\
\hline VS2 & ns & ns & $\begin{array}{l}p=0.018 \\
(t=2.77)\end{array}$ & ns & ns & ns \\
\hline
\end{tabular}

$p$ Values are corrected for multiple comparisons

ns, Not significant; Pre-PMd, dorsal pre-premotor cortex; PMd, dorsal premotor cortex; SMA, supplementary motor area; pre-SMA, pre-supplementary motor area. 

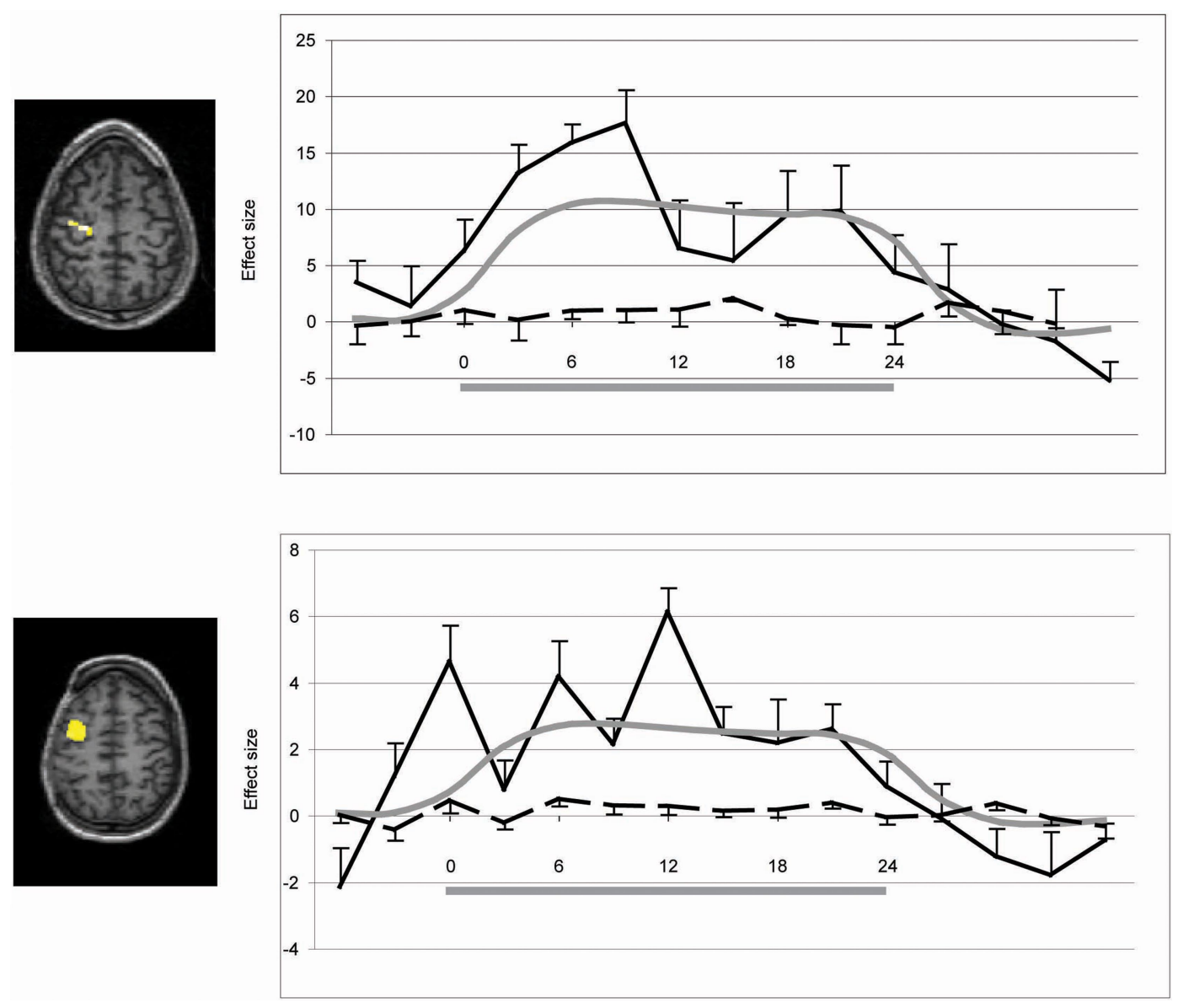

FIGURE $\mathbf{5}$ | Time course of ROI activity for left premotor cortex for VS1 and VS2. The gray horizontal bar shows when the commands were delivered. The solid black line corresponds to the "right hand vs. left hand" contrast, whereas the dashed line corresponds to the "left hand vs. right hand "contrast. Standard errors are shown for each contrast." The gray line corresponds to the model's HRF.

the clinical assessment. Consciousness is assessed by measuring an active response to a command. Given that the paradigm we used required understanding of verbal instructions, only those patients with demonstrated preserved auditory processing for basic speech characteristics could be included. This reduced the original sample size from 24 to 5 . Two out of these five patients showed signs of residual conscious processing. Thus, the small number is greatly explained by the relatively low proportion of patients with preserved speech processing after traumatic brain injury (Coleman et al., 2009), not by the low detection rate of our method. Considering these limitations, the identification of two patients with potential for recovery is of clinical relevance.

We have previously used fMRI to assess residual conscious awareness in clinically diagnosed VS patients (Owen et al., 2006; Monti et al., 2010). Nevertheless, the high cognitive demands associated with that experimental paradigm (Guillot and Collet, 2005) may restrict its application to high functioning patients in MCS or patients with relatively spared cognitive processing. Here, we showed that signs of purposeful behavior can be inferred with a much simpler motor paradigm based on movement preparatory activity in the absence of overt action. The overall pattern of brain activity observed for VS1 and VS2 was greater and more extended than that found for the other three patients. This is consistent with the observation that MCS patients exhibit a more extended pattern of brain activity than VS patients in a variety of tasks (Boly et al., 2004; Schnakers et al., 2008; Bekinschtein et al., 2009). The fact that behavioral re-assessment of VS1 3 years after fMRI scanning was compatible with a diagnosis of MCS, further suggests that signs of voluntary behavior may reflect residual conscious awareness. Importantly however, there was no evidence of volition from the first behavioral evaluation. Unfortunately, we were unable to carry out a full behavioral 2-year follow-up of VS2.

From a methodological point of view, the innovation of this paradigm lies in the use of a simple motor task that does not require high-level cognitive processing and therefore could be used to detect conscious awareness in a presumably larger VS patient population. We believe that functional imaging techniques should be used as a complementary test to confirm conscious awareness in patients who have already been through a comprehensive clinical assessment and several physiological tests using affordable and portable methods such as EMG and EEG to assess basic auditory, perception, and speech (Coleman et al., 2009b). In this manner fMRI could 
serve as an additional tool to detect signs of voluntary movement which may reflect residual conscious awareness in those patients that have been insensitive to other methods. It is important to keep in mind, however, that fMRI is limited in its interpretation: whereas positive results such as those shown here, are very informative and often straightforward to interpret, negative results are ambiguous as they can reflect true negative results, a drop in signal intensity, changes in wakefulness, lack of attention, etc.

In conclusion, we have used a simple motor preparation paradigm to objectively detect signs of purposeful behavior that were not evident from the clinical assessment in two of five patients who were presumed to be vegetative (and therefore unconscious). The current study argues for the use of functional MRI in the assessment and diagnosis of disorders of consciousness following severe brain injury. We believe that neurorehabilitation centers could benefit greatly from incorporating these and other objective tools such as electromyographic (Bekinschtein et al., 2008, 2009b) or electroencephalographic (e.g., Schnakers et al., 2008; Bekinschtein et al., 2009a) methods to identify those patients with potential for recovery. Moreover, recent advances in Brain Computer Interface paradigms, showing high accuracy of communication through EEG signals after

\section{REFERENCES}

Adams, J. H., Graham, D. I., and Jennett, B. (2000). The neuropathology of the vegetative state after an acute brain insult. Brain 123, 1327-1338.

American Neurophysiology Society. (2006). Guideline 9C: guidelines on short-latency auditory evoked potentials. Am. J. Electroneurodiagnostic Technol. 46, 275-286.

Andersen, R. A., and Buneo, C. A. (2002). Intentional maps in posterior parietal cortex. Annu. Rev. Neurosci. 25, 189-220.

Andrews, K., Murphy, L., Munday, R., and Littlewood, C. (1996). Misdiagnosis of the vegetative state: retrospective study in a rehabilitation unit. $B M$ ) 313, 13-16.

Bekinschtein, T., Leiguarda, R., Armony, J., Owen, A., Carpintiero, S., Niklison, J., Olmos, L., Sigman, L., and Manes, F. (2004). Emotion processing in the minimally conscious state. J. Neurol. Neurosurg. Psychiatry 75, 788.

Bekinschtein, T. A. (2006). Cognitive Processes in the Vegetative and Minimally Conscious State. Thesis, University of Buenos Aires, Buenos Aires.

Bekinschtein, T. A., Coleman, M. R., Niklison, J., Pickard, J. D., and Manes, F. F. (2008). Can electromyography objectively detect voluntary movement in disorders of consciousness? J. Neurol. Neurosurg. Psychiatry 79, 826-828.

Bekinschtein, T. A., Dehaene, S., Rohaut, B., Taddel, F., Cohen, L., and Naccache, L. (2009a). Neural signatures of the conscious processing of auditory reg- ularities. Proc. Natl. Acad. Sci. U.S.A. 106, 1672-1677.

Bekinschtein, T.A., Shalom, D. E., Forcato, C., Herrera, M., Coleman, M. R., Manes, F. F., and Sigman, M. (2009b). Classical conditioning in the vegetative and minimally conscious state. Nat. Neurosci. 12, 1343-1349.

Blankertz, B., Losch, F., Krauledat, M., Dornhege, G., Curio, G., and Müller, K. R. (2008). The Berlin Brain-Computer Interface: accurate performance from first-session in BCI-naïve subjects. IEEE Trans. Biomed. Eng. 55, 2452-2462.

Boly, M., Faymonville, M. E., Peigneux, P., Lambermont, B., Damas, P., Del Fiore, G., Degueldre, C., Franck, G., Luxen, A., Lamy, M., Moonen, G., Maquet, P., and Laureys, S. (2004). Auditory processing in severely brain injured patients: differences between the minimally conscious state and the persistent vegetative state. Arch. Neurol. 2, 233-238.

Boly, M., Faymonville, M. E., Schnakers, C., Peigneux, P., Lambermont, B., Phillips, C., Lancellotti, P., Luxen, A., Lamy, M., Moonen, G., Maquet, P., and Laureys, S. (2008). Perception of pain in the minimally conscious state with PET activation: an observational study. Lancet Neurol. 11, 1013-1020.

Brett, M., Anton, J.-L., Valabregue, R., and Poline, J.-B. (2002). "Region of interest analysis using an SPM toolbox," in Paper Presented at the 8th International Conference on Functional Mapping of the Human Brain, Sendai.

Childs, N. L., Mercer, W. N., and Childs, H.W. (1993).Accuracy of diagnosis of

very little training (Blankertz et al., 2008) may be adapted in the future to be used in patients with disorders of consciousness. A multimodal approach is desirable to minimize misdiagnosis of these patients as a result of a functional deficit in a particular perceptual domain, in the production of movement or due to low sensitivity of the tests performed (Coleman et al., 2009).

\section{ACKNOWLEDGMENTS}

We thank the care homes and rehabilitation centers in Argentina for their contribution and we specially thank all of the participants. We also thank Mathew Brett for technical support and Jorge Niklison for assisting with the data acquisition and patient assessment. This study was undertaken during the course of Tristan Andres Bekinschtein's Ph.D. This work was supported by an Antorchas Foundation Student Grant and a Marie Curie Fellowship to Tristan Andres Bekinschtein, a StartUp Grant to Facundo Francisco Manes and a Return Home International Brain Research Organization (IBRO) Grant to Valeria Della-Maggiore. The funding sources had no role in the study design, data collection, data analysis, data interpretation, or writing of this report. All authors had full access to all the data in the study and had final responsibility for the decision to submit for publication.

persistent vegetative state. Neurology 43, 1465-1467.

Coleman, M. R., Bekinschtein, T., Monti, M. M., Owen, A. M., and Pickard, J. D. (2009a). A multimodal approach to the assessment of patients with disorders of consciousness. Prog. Brain Res. 177C, 231-248.

Coleman, M. R., Davis, M. H., Rodd, J. M., Robson, T., Ali, A., Owen, A. M., and Pickard, J. D. (2009b). Towards the routine use of brain imaging to aid the clinical diagnosis of disorders of consciousness. Brain 132, 2541-2552.

Coleman, M. R., Rodd, J. M., Davis, M. H., Johnsrude, I. S., Menon, D., Pickard, J. D., and Owen, A. M. (2007). Do vegetative patients retain aspects of language comprehension? Evidence from fMRI. Brain 130, 2494-2507.

Crammond, D. J., and Kalaska, J. F. (1994) Modulation of preparatory neuronal activity in dorsal premotor cortex due to stimulus-response compatibility. J. Neurophysiol. 71, 1281-1284.

Crammond, D. J., and Kalaska, J. F. (2000). Prior information in motor and premotor cortex: activity during the delay period and effect on premovement activity. J. Neurophysiol. 84, 986-1005.

Davis, M.H., Coleman, M. R., Absalom, A R., Rodd, J. M., Johnsrude, I. S., Matta, B. F., Owen, A. M., and Menon, D. K. (2007). Dissociating speech perception and comprehension at reduced levels of awareness. Proc. Natl. Acad. Sci. U.S.A. 104, 16032-16037.

Fernandez-Espejo, D., Bekinschtein, T.A., Monti, M. M., Pickard, J. D., Junque, C., Coleman, M. R., and Owen, A. M.
(2011). Diffusion weighted imaging distinguishes the vegetative state from the minimally conscious state. Neuroimage 54, 103-112.

Galton, C. J., Gomez-Anson, B., Antoun, N., Scheltens, P., Patterson, K., Graves, M., Sahakian, B. J., and Hodges, J. R. (2001). Temporal lobe rating scale: application to Alzheimer's disease and frontotemporal dementia. J. Neurol. Neurosurg. Psychiatry 70, 165-173.

Giacino, J. T., Ashwal, S., Childs, N., Cranford, R., Jennett, B., Katz, D. I., Kelly, J. P., Rosenberg, J. H., Whyte, J., Zafonte, R.D., and Zasler, N.D. (2002). The minimally conscious state: definition and diagnostic criteria. Neurology 58, 349-353.

Giacino, J. T., Kalmar, K., and Whyte, J. (2004). The JFK coma recovery scalerevised: measurement characteristics and diagnostic utility. Arch. Phys. Med. Rehabil. 85, 2020-2029.

Gill-Thwaites, H. (2006). Lotteries, loopholes and luck: misdiagnosis in the vegetative state patient. Brain Inj. 20, 1321-1328.

Guillot, A., and Collet, C. (2005). Contributions from neurophysiological and neuropsychological methods to the study of motor imagery. Brain Res. Rev. 50, 387-397.

Kotchoubey, B., Lang, S., Mezger, G., Schmalohr, D., Schneck, M., Semmler, A., Bostanov, V., and Birbaumer, N. (2005). Information processing in severe disorders of consciousness: vegetative state and minimally conscious state. Clin. Neurophysiol. 116, 2441-2453. 
Laureys, S., Faymonville, M. E., Peigneux, P., Damas, P., Lambermont, B., Del Fiore, G., Degueldre, C., Aerts, J., Luxen, A., Franck, G., Lamy, M., Moonen, G., and Maquet, P. (2002). Cortical processing of noxious somatosensory stimuli in the persistent vegetative state. Neuroimage 17, 732-741.

Laureys, S., Owen, A. M., and Schiff, N. D. (2004). Brain function in coma, vegetative state, and related disorders. Lancet Neurol. 3, 537-546.

Menon, D. K., Owen, A. M., Williams, E. J., et al. (1998). Cortical processing in persistent vegetative state. Lancet 352, 200.

Monti, M. M., Vanhaudenhuyse, A., Coleman, M. R., Boly, M., Pickard, J. D., Tshibanda, L., Owen, A. M., and Laureys, S. (2010). Willful modulation of brain activity in disorders of consciousness. N. Engl. J. Med. 362, 579-589.

Multi-Society Task Force on PVS. (1994). Medical aspects of the persistent vegetative state: parts I and II. N. Engl. J. Med. 330, 1499-1508, 1572-1579.

Owen, A. M., and Coleman, M. R. (2008). Functional neuroimaging of the vegetative state. Nat. Rev. Neurosci. 9, 235-243.
Owen, A. M., Coleman, M. R., Boly, M., Davis, M. H., Laureys, S., and Pickard, J.D. (2006). Detecting awareness in the vegetative state. Science 313, 1402.

Owen, A. M., Menon, D. K., Johnsrude, I. S., Bor, D., Scott, S. K., Manly, T., Williams, E. J., Mummery, C., and Pickard, J. D. (2002). Detecting residual cognitive function in persistent vegetative state. Neurocase 8, 394-403.

Passingham, R. E. (1985). Premotor cortex: sensory cues and movement. Behav. Brain Res. 18, 175-185.

Picard, N., and Strick, P. L. (2001). Imaging the premotor areas. Curr. Opin. Neurobiol. 11, 663-672.

Poldrack, R. A., Fletcher, P. C., Henson, R. N., Worsley, K. J., Brett, M., and Nichols, T.E. (2008). Guidelines for reporting an fMRI study. Neuroimage 40, 409-414.

Portas, C. M., Krakow, K., Allen, P., Josephs, O., Armony, J. L., and Frith, C. D. (2000). Auditory processing across the sleep-wake cycle: simultaneous EEG and fMRI monitoring in humans. Neuron 3, 991-999.

Royal College of Physicians. (2003). The Vegetative State: Guidance on Diagnosis and Management (Report of a Working Party). London: Royal College of Physicians.
Schiff, N., Ribary, U., Moreno, D., Beattie, B., Kronberg, E., Blasberg, R., et al. (2002). Residual cerebral activity and behavioural fragments in the persistent vegetative state. Brain 125 , 1210-1234.

Schnakers, C., Perrin, F., Schabus, M., Majerus, S., Ledoux, D., Damas, P., Boly, M., Vanhaudenhuyse, A., Bruno, M. A., Moonen, G., and Laureys, S. (2008). Voluntary brain processing in disorders of consciousness. Neurology 71, 1614-1620.

Scott, S. K., and Johnsrude, I. S. (2003). The neuroanatomical and functional organization of speech perception. Trends Neurosci. 26, 100-107.

Thoenissen, D., Zilles, K., and Toni, I. (2002). Differential involvement of parietal and precentral regions in movement preparation and motor intention. J. Neurosci. 22, 9024-9034.

Toni, I., Schluter, N. D., Josephs, O., Friston, K., and Passingham, R. E. (1999). Signal-, set- and movementrelated activity in the human brain: an event-related fMRI study. Cereb. Cortex 9, 35-49.

Vuilleumier, P., and Assal, G. (1995). Lesions of the corpus callosum and syndromes of interhemispheric disconnection of traumatic origin. Neurochirurgie 41, 98-107.

Wise, S. P., Weinrich, M., and Mauritz, K. H. (1983). Motor aspects of cuerelated neuronal activity in premotor cortex of the rhesus monkey. Brain Res. 260, 301-305.

Conflict of Interest Statement: The authors declare that the research was conducted in the absence of any commercial or financial relationships that could be construed as a potential conflict of interest.

Received: 23 November 2009; accepted: 08 January 2011; published online: 27 January 2011.

Citation: Bekinschtein TA, Manes FF, Villarreal $M$, Owen $A M$ and DellaMaggiore V (2011) Functional imaging reveals movement preparatory activity in the vegetative state. Front. Hum. Neurosci. 5:5. doi: 10.3389/fnhum.2011.00005

Copyright (C) 2011 Bekinschtein, Manes, Villarreal, Owen and Della-Maggiore. This is an open-access article subject to an exclusive license agreement between the authors and Frontiers Media SA, which permits unrestricted use, distribution, and reproduction in any medium, provided the original authors and source are credited. 


\section{APPENDIX \\ PATIENTS' ASSESSMENTS AND DESCRIPTION}

Patient VS1 had a severe head injury following a motorbike accident. He remained in coma for 2 weeks progressing to VS after sedation withdrawal. The patient was admitted in our facility 16 months after the accident with a CRS-R score of 8 , and was scanned 2 weeks later (CRS-R of 8). Clinical assessment showed eye fixation and inconsistent orienting to sounds to the right side. He also occasionally groaned when repositioned in the bed or during blood extractions but this behavior was not reproducible. The structural MRI image showed mild cortical and severe callosal and hippocampal atrophy with diffuse axonal injury and small diencephalic infarcts. In the electrophysiological evaluation, MMN wave was present in a classic passive auditory (oddball) event-related potentials paradigm (20 electrodes, $250 \mathrm{~Hz}$, ATI systems, sine wave tones presented binaurally, 15\% rare tones, $1000 \mathrm{~ms}$ ISI). The last thorough behavioral assessment carried out 34 months after the fMRI study revealed a CRS-R score of 11 and showed a wider behavioral portfolio: the patient was able to follow a mirror in front of his eyes, orient his head and eyes to sounds and localize noxious stimuli on the left arm crossing the body midline with his right arm, thereby fulfilling the diagnostic criteria for MCS.

Patient VS2 was hospitalized after a car accident. The patient was admitted in our facility 3 months after the accident. The clinical assessment yielded very low scores on standard behavioral scales, with a few spared reflexes; his initial CRS-R score was 5. After a change in medication (sedatives were reduced and methylphenidate was administered) he started to show some inconsistent visual fixation. The MMN response in an event-related potential auditory task revealed some degree of cortical integrative processing (same paradigm described for VS1). The structural MRI image showed left temporofrontal contusion, right parietal and internal capsule contusion, enlarged ventricles and mild frontal atrophy. At the time of the fMRI assessment, 5 months after the accident, the patient showed a score of 7 in the CRS-R, with spontaneous eye opening, oral reflexive movements, preserved limb withdrawal reflex, and spared auditory and visual reflexes. The last full behavioral testing performed at the hospital ( 5 months after the scan) showed vocalization (CRS-R score of 8). Unfortunately, we were unable to carry out a full behavioral 2-year follow-up assessment of VS2 given that he/she has moved back to the south of the country. A local neurologist who carried out the standard neurological assessment informed no changes up to date.

Patient VS3 had an open head injury with a long delay before medical attention leading to additional anoxic insults. The patient was admitted in our facility 20 months after the head injury. During the full evaluation she showed bruxism and saliva swallowing episodically and systematic chewing when objects or food were placed in her mouth with automatic swallowing preserved. Noxious stimuli evoked flexor or extensor postural reflexes; auditory (startle), threat and olfactory reflexes were preserved. She displayed teeth clenching and facial flushing when spontaneous movements were restrained, when her eyes were covered or when her face was touched. Structural MRI image identified severe cerebral atrophy with dilated ventricles, wide cerebral sulci and diffuse axonal injury. Passive auditory ERP showed mildly delayed
MMN responses to infrequent tones. The most recent thorough behavioral assessment obtained 15 months after the fMRI study, indicated that the patient remained in vegetative state despite her rich behavioral portfolio.

Patient VS4 fell from a horse suffering a severe traumatic head injury and brain swelling. Three weeks later, a shunt was inserted to allow CSF drainage. The patient was admitted in our facility 5 months after the accident. During the full evaluation she showed preserved reflexes (including swallowing) and responded to nociceptive stimuli to the right side of her body only. She also showed facial expressions but they were not linked to events or stimuli. The structural MRI image revealed cortical and subcortical atrophy and bilateral ventriculomegaly. The most recent thorough behavioral assessment obtained 7 months after the fMRI study, indicated that the patient remained in vegetative state.

Patient VS5 suffered severe head trauma following a motor vehicle accident and postoperative ischemic events. The patient was admitted in our facility 3 months after the accident. The clinical examination showed low behavioral scores. After pro-dopaminergic medication, the behavioral score increased to the upper limit of the VS category: the patient could fixate briefly on a person and on a bright object if presented in his field of view; he also showed mechanical vocalization, such as yawning and sighing. The patient's structural MRI image revealed mild callosal and cortical atrophy, diencephalic contusion, a left temporoinsular contusion hemorrhage and a semioval center contusion. The last clinical assessment obtained 23 months after the fMRI study showed no changes from the previous examination except for occasional crying. No causal relationship could be established between external events and crying following the assessment.

\section{VALIDATION OF THE EXPERIMENTAL PROTOCOL: ACTIVATION IN NORMAL VOLUNTEERS}

Three normal subjects (male, 23, 24, and 28 y.o.) were scanned with the same fMRI protocol to detect signs of movement preparation as part of a large fMRI validation study (Bekinschtein, 2006). The data was analyzed following the same procedure used for the patients (fixed-effects approach). Whole-brain statistical analysis showed large clusters of activity when comparing left vs. right-hand commands and right vs. left-hand commands covering portions of the somatosensory and motor cortex (hand area), dorsal premotor and pre-premotor cortex. SMA and pre-SMA activity was also present in two of three subjects for the right vs. left-hand commands. Figure A1 shows the overlapped pattern of brain activity for the right vs. lefthand command contrast for each of the three subjects superimposed on the regions of interest depicted in Figure 1 (single subject analysis). Only the results from this contrast are shown in the figure because it yielded specific activity changes in VS1 and VS2. The ROI analysis revealed significant brain activity for all three subjects for each of the ROI in both left vs. right and right vs. left-hand command comparisons (for details on the contribution of each subject to the spatial pattern depicted in Figure A1; see Table A1). It is important to take into consideration however, that the pattern of activation detected in the controls not only reflected motor preparation but also movement execution. This is an intrinsic limitation of having scanned patients and normal subjects while performing the same 
task. Changing the task, however, by separating preparation from action would have required an event-related design, which would have been difficult to compare to the patients' data.

\section{ANALYSIS OF SIGNAL LOSS AREAS IN THE SPM MASKS}

In order to characterize the profile of signal loss in patients VS1 and VS2, and to estimate to what extent it may have influenced our results, we superimposed the SPM activity maps for right vs. left movement contrast over their SPM masks (Figure A2). SPM masks images are $3 \mathrm{D}$ space binary data representing the voxels defined as the search space for the statistical analysis, a mask is an image indicating which voxels were included in the analysis. Mask are highly susceptible to motion and changes in the signal due to lesioned tissue. On the analysis we confirmed that most brain regions in motor/premotor areas were included in the masks. Only a small lateral portion of the left dorsal pre-premotor cortex was affected due to signal inhomogeneities from focal hemorrhages in VS2 and VS5. In terms of activations we found no activation blobs near areas with signal drop (mostly lesioned areas) except for an SMA activity cluster in VS2 that fell on a partial volume of the upper slice of the $\mathrm{T} 2{ }^{*}$-weighted volume. The signal drop areas revealed by the masks do not co-localize with the activation clusters. Figure A3 shows the results for the contrast right hand vs. rest for patients VS3, VS4, and VS5 overlaid on their SPM mask. These three patients did not show significant activation in the right vs. left hand contrast (or left vs. right), giving the impression that they were truly vegetative.

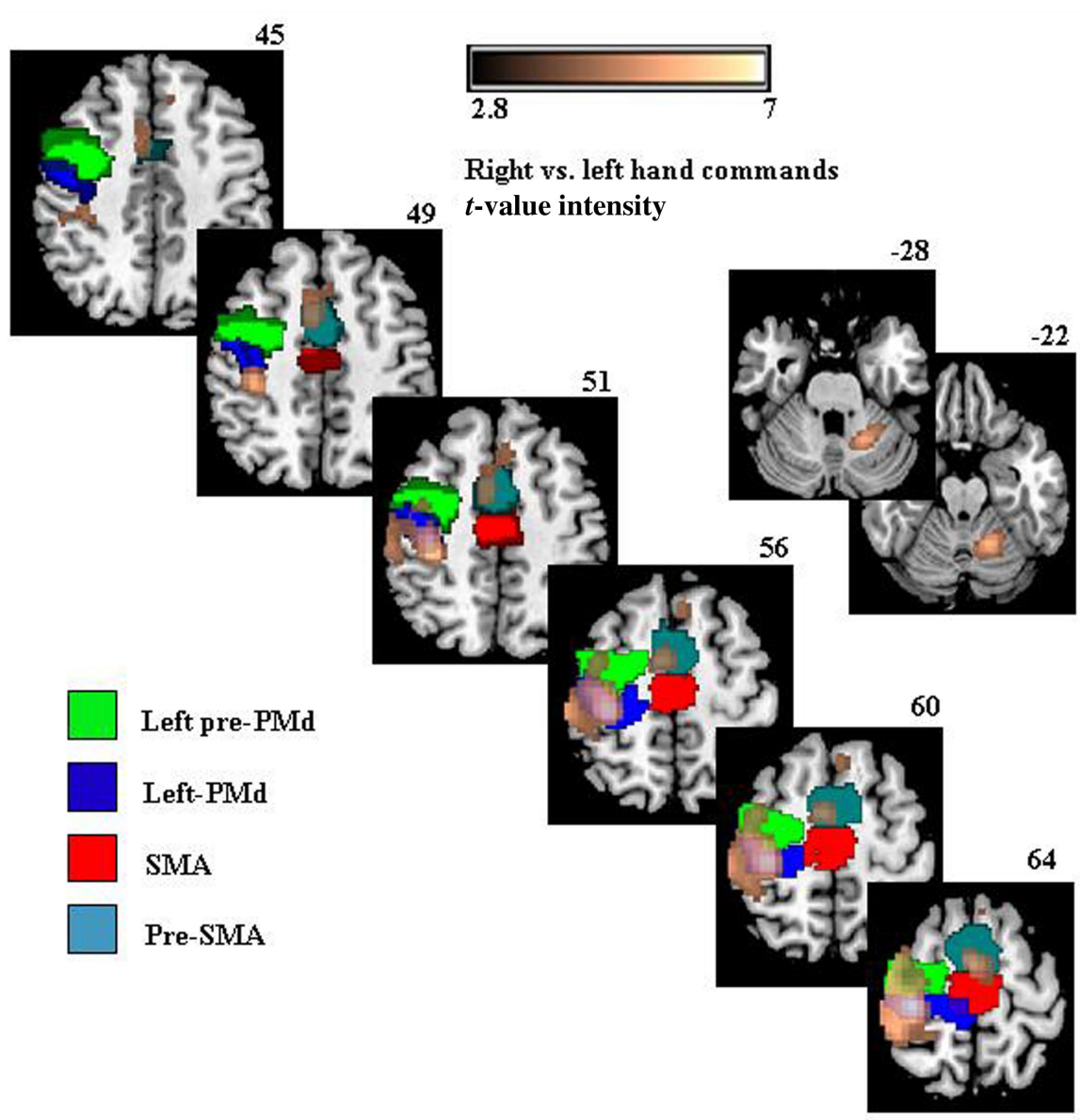

FIGURE A1 | Command induced activity in premotor cortex in healthy volunteers. Activity (in brown) corresponds to the results for the right vs. left-hand command contrast for each of the three control subjects (single subject fixed effects). $t$ Maps are superimposed on the ROI map depicted in Figure 1 and overlaid on axial slices of the 152-MNIT1 template. See Table A1 for details on the contribution of each subject to this spatial pattern. 
Table A1 | $t$ Values for contrasts left vs. right and right vs. left-hand commands for the ROls in all three normal volunteers.

\begin{tabular}{lllllll}
\hline \multicolumn{1}{c}{ R-Pre-PMd } & R-PMd & L-Pre-PMd & L-PMd & SMA & Pre-SMA \\
\hline \multicolumn{7}{l}{ LEFT VS. RIGHT-HAND COMMANDS } \\
NV1 & $4.33^{* *}$ & $5.67^{* *}$ & $\mathrm{~ns}$ & $\mathrm{~ns}$ & $\mathrm{~ns}$ & $\mathrm{~ns}$ \\
NV2 & $5.16^{* *}$ & $6.42^{* * *}$ & $\mathrm{~ns}$ & $\mathrm{~ns}$ & $\mathrm{~ns}$ & $\mathrm{~ns}$ \\
NV3 & $3.11^{*}$ & $7.03^{* * *}$ & $\mathrm{~ns}$ & $\mathrm{~ns}$ & $\mathrm{~ns}$ & $\mathrm{~ns}$ \\
RIGHT VS. LEFT-HAND COMMANDS & & & \\
NV1 & $\mathrm{ns}$ & $\mathrm{ns}$ & $3.79^{* *}$ & $6.78^{* * *}$ & $2.77^{*}$ & $4.56^{* *}$ \\
NV2 & $\mathrm{ns}$ & $\mathrm{ns}$ & $5.13^{* *}$ & $7.72^{* * *}$ & $2.83^{*}$ & $6.14^{* * *}$ \\
NV3 & $\mathrm{ns}$ & $\mathrm{ns}$ & $5.83^{* * *}$ & $7.95^{* * *}$ & $\mathrm{~ns}$ & $\mathrm{~ns}$ \\
\hline
\end{tabular}

$N V$, normal volunteer, ${ }^{*} p<0.05,{ }^{*} p<0.005,{ }^{*}{ }^{*} p<0.001$, corrected for multiple comparisons; ns, not significant; Pre-PMd, dorsal pre-premotor cortex; PMd, dorsal premotor cortex; SMA, supplementary motor area; pre-SMA pre-supplementary motor area.
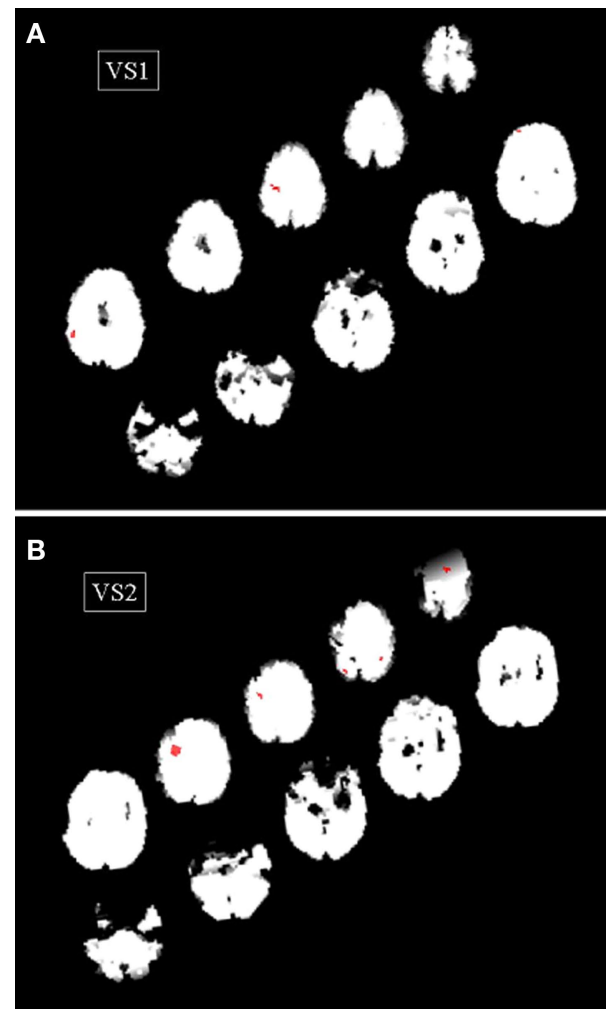

FIGURE A2 | Results of the right vs. left movement contrast (red) superimposed on the original SPM masks for VS1 (A) and VS2 (B)
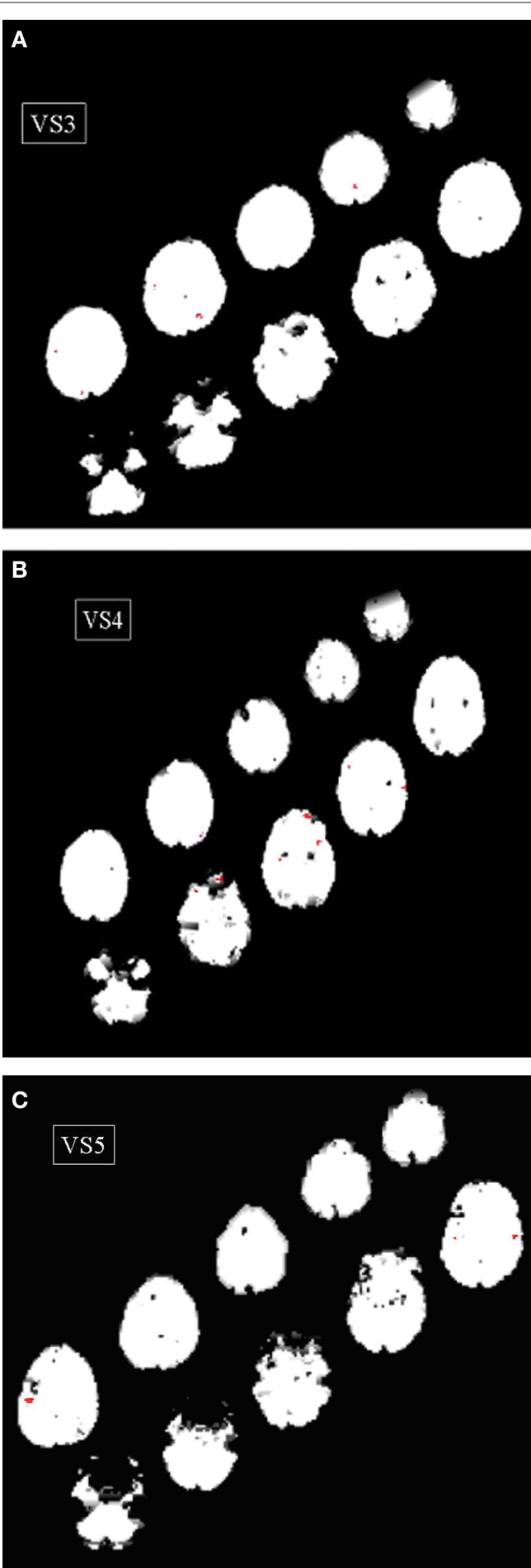

FIGURE A3 | Results of the right vs. rest movement contrast (red) superimposed on the original SPM masks for VS3 (A), VS4 (B), and VS5 (C) 